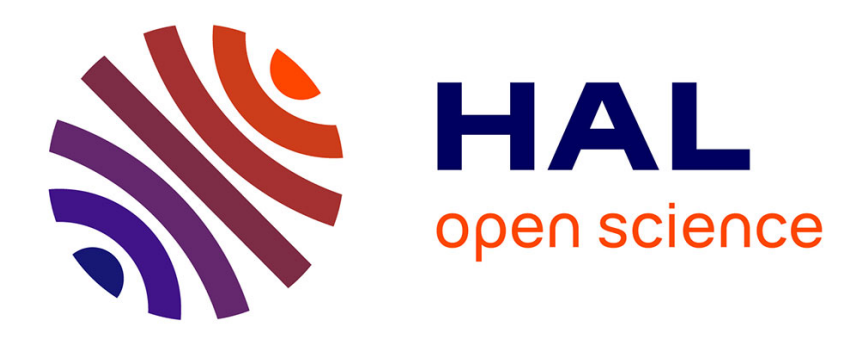

\title{
Efficiently Summarizing Data Streams over Sliding Windows
}

\author{
Nicoló Rivetti, Yann Busnel, Achour Mostefaoui
}

\section{To cite this version:}

Nicoló Rivetti, Yann Busnel, Achour Mostefaoui. Efficiently Summarizing Data Streams over Sliding Windows. IEEE 14th International Symposium on Network Computing and Applications, Sep 2015, Cambrige, United States. pp.151 - 158, 10.1109/NCA.2015.46 . hal-01535693

\section{HAL Id: hal-01535693 https://hal.science/hal-01535693}

Submitted on 9 Jun 2017

HAL is a multi-disciplinary open access archive for the deposit and dissemination of scientific research documents, whether they are published or not. The documents may come from teaching and research institutions in France or abroad, or from public or private research centers.
L'archive ouverte pluridisciplinaire HAL, est destinée au dépôt et à la diffusion de documents scientifiques de niveau recherche, publiés ou non, émanant des établissements d'enseignement et de recherche français ou étrangers, des laboratoires publics ou privés. 


\section{Efficiently Summarizing Data Streams over Sliding Windows}

\author{
Nicoló Rivetti \\ LINA / Université de Nantes, \\ Nantes, France \\ Nicolo.Rivetti@univ-nantes.fr
}

\author{
Yann Busnel \\ Crest (Ensai) / Inria, \\ Rennes, France \\ Yann.Busnel@ensai.fr
}

\author{
Achour Mostefaoui \\ LINA / Université de Nantes, \\ Nantes, France \\ Achour.Mostefaoui@univ-nantes.fr
}

\begin{abstract}
Estimating the frequency of any piece of information in large-scale distributed data streams became of utmost importance in the last decade (e.g., in the context of network monitoring, big data, etc.). If some elegant solutions have been proposed recently, their approximation is computed from the inception of the stream. In a runtime distributed context, one would prefer to gather information only about the recent past. This may be led by the need to save resources or by the fact that recent information is more relevant.

In this paper, we consider the sliding window model and propose two different (on-line) algorithms that approximate the items frequency in the active window. More precisely, we determine a $(\varepsilon, \delta)$-additive-approximation meaning that the error is greater than $\varepsilon$ only with probability $\delta$. These solutions use a very small amount of memory with respect to the size $N$ of the window and the number $n$ of distinct items of the stream, namely, $O\left(\frac{1}{\varepsilon} \log \frac{1}{\delta}(\log N+\log n)\right)$ and $O\left(\frac{1}{\tau \varepsilon} \log \frac{1}{\delta}(\log N+\log n)\right)$ bits of space, where $\tau$ is a parameter limiting memory usage. We also provide their distributed variant, i.e., considering the sliding window functional monitoring model. We compared the proposed algorithms to each other and also to the state of the art through extensive experiments on synthetic traces and real data sets that validate the robustness and accuracy of our algorithms.
\end{abstract}

\section{INTRODUCTION AND RELATED WORK}

In large distributed systems, it is most likely critical to gather various aggregates over data spread across the large number of nodes. This can be modelled by a set of nodes, each observing a stream of items. These nodes have to collaborate to continuously evaluate a given function over the global distributed stream. For instance, current network management tools analyze the input streams of a set of routers to detect malicious sources or to extract user behaviors [1], [2]. The main goal is to evaluate such functions at the lowest cost in terms of the space used at each node, as well as minimizing the update and query time. The solutions proposed so far are focused on computing functions or statistics using $\varepsilon$ or $(\varepsilon, \delta)$-approximations in polylogarithmic space over the size $m$ of the stream and the number $n$ of its distinct items.

In the data streaming model, many functions have been studied such as the estimation of the number of distinct data items in a stream [3], [4], the frequency moments [5], the most frequent data items [6], the frequency estimation [7], [8] or information divergence over streams [1]. Cormode et al. [9]

This work was partially funded by the French ANR project SocioPlug (ANR 13-INFR-0003), and by the DeSceNt project granted by the Labex CominLabs excellence laboratory (ANR-10-LABX-07-01). propose solutions for frequency moments estimation in the functional monitoring model. In most applications, computing such a function from the inception of a distributed stream is useless [10]. Only the most recent past may be relevant meaning that the function has to be evaluated on part of the stream captured by a window of a given size ( say $N$ ) that will slide over time. Datar et al. [10] introduced the sliding window concept in the data streaming model presenting the exponential histogram algorithm that provides an $\varepsilon$-approximation for basic counting. Gibbons and Tirthapura [11] presented an algorithm matching the results of [10] based on the wave data structures requiring constant processing time and providing some extensions for distributed streams. Arasu and Manku [12] studied the problem of $\varepsilon$-approximating counts over sliding windows, presenting both deterministic and randomized solutions achieving respectively $O\left(\frac{1}{\varepsilon} \log ^{2} \frac{1}{\varepsilon}\right)$ and $O\left(\frac{1}{\varepsilon} \log \frac{\varepsilon}{\delta}\right)$ space complexity. In this model there are also works on variance [13], quantiles [12] and frequent items [14]. Merging both models, [15] provides an optimal solution for the heavy hitters problem in the sliding window functional monitoring model.

In this paper, we tackle the frequency estimation problem in the sliding window model. Whatever is the model, this problem cannot be reduced to the heavy hitters (frequent items) problem and approximate counts. Indeed, having the frequency estimation of items allows to determine frequent element but the converse does not hold. Moreover, using little memory (low space complexity) implies some kind of data aggregation. If the number of counters is less than the number of different items then necessarily each counter encodes the occurrences of more than one item. The problem is then how to slide the window to no more keep track of the items that exited the window and how to introduce new items. As a consequence, our work cannot be compared to [14], [16]. To our knowledge the only work that tackles a similar problem is [17]. Their proposal, named ECM-sketches, consists in a compact structure combining some state-of-the-art sketching techniques for data stream summarization, with sliding window synopses.

We extend the well-known algorithm for frequency estimation, namely the COUNT-MIN sketch [8], in a windowed version. We propose our approach in two steps, two first naive and straightforward algorithms called PERFECT and SIMPLE followed by two more sophisticated ones called PROPORTIONAL windowed and SPLITTER windowed algorithms. Then, we compare their respective performances together with the ECMsketches solution, proposed in [17]. 
This paper is composed of 5 Sections. Section II describes the computational model and some necessary background. In Section III, after two naive first step algorithms, we propose two novel $(\varepsilon, \delta)$-additive-approximations, achieving respectively $O\left(\frac{1}{\varepsilon} \log \frac{1}{\delta}(\log N+\log n)\right)$ and $O\left(\frac{1}{\tau \varepsilon} \log \frac{1}{\delta}(\log N+\log n)\right)$ bits $^{1}$ of space, where $\tau$ is an additional parameter limiting memory usage (see Section III-D). Section III-E and Section III-F present respectively the distributed variant and the timebased sliding windows extention. The efficiency of the three algorithms and the algorithm proposed in [17] are analyzed and Section IV presents an extended performance evaluation of the estimation accuracy of our algorithms, with both synthetic traces and real data sets, inspired by [18].

\section{PRELIMINARIES AND BACKGROUND}

\section{A. Data Streaming Model}

We present the computation model under which we analyze our algorithms and derive bounds: the data streaming model [19]. We consider a massively long input stream $\sigma$, that is, a sequence of elements $\left\langle a_{1}, a_{2}, \ldots, a_{m}, \ldots\right\rangle$ called samples. Samples are drawn from a universe $[n]=\{1,2, \ldots, n\}$ of items. The size of the universe (or number of distinct items) of the stream is $n$. This sequence can only be accessed in its given order (no random access). The problem to solve can be seen as a function $\phi$ evaluated on a sequence of items prefix of size $m$ of a stream $\sigma$ under memory constraints. For example if the function $\phi$ represents the most frequent item then the function $\phi$ applied to the first $m$ items of the stream returns the most frequent item among these $m$ first samples.

In order to reach these goals, we rely on randomized algorithms that implement approximations of the desired function $\phi$. Namely, such an algorithm $\mathcal{A}$ evaluates the stream in a single pass (on-line) and continuously. It is said to be an $(\varepsilon, \delta)$-additive-approximation of the function $\phi$ on a stream $\sigma$ if, for any prefix of size $m$ of items of the input stream $\sigma$, the output $\hat{\phi}$ of $\mathcal{A}$ is such that $\mathbb{P}\{|\hat{\phi}-\phi|>\varepsilon C\}<\delta$, where $\varepsilon, \delta>0$ are given as precision parameters and $C$ is an arbitrary constant. The parameter $\varepsilon$ represents the precision of the estimation of the approximation. For instance $\varepsilon=0.1$ means that the additive error is less than $10 \%$ and $\delta=0.01$ means that this approximation will not be satisfied with a probability less than $1 \%$.

On the other hand, as explained in the Introduction, we are only interested in the recent past. This is expressed by the fact that when the function $\phi$ is evaluated, it will be only on the $N$ more recent items among the $m$ items already observed, that is, the sliding window model formalized by Datar et al. [10]. In this model, samples arrive continuously and expire after exactly $N$ steps. A step corresponds to a sample arrival, i.e., we consider count-based sliding windows. The challenge consists in achieving this computation in sub-linear space. When $N$ is set to the maximal value of $m$, the sliding window model boils down to the classical model. The supplemental problem brought by a sliding window resides in the fact that when a prefix of a stream is summarized we lose the temporal information related to the different items making the exclusion of the most ancient items non trivial with little memory.

\footnotetext{
${ }^{1}$ For the sake of clarity, we will use the notation log to denote the logarithm in base 2 for the rest of this paper.
}

\section{B. Vanilla Count-Min Sketch}

The problem we tackle in this paper is the frequency estimation problem. In a stream, each item appears a given number of times that allows to define its frequency. The function that defines this problem returns a frequency vector $\mathbf{f}=\left(f_{1}, \ldots, f_{n}\right)$ where $f_{j}$ represents the number of occurrences of item $j$ in the portion of the input stream $\sigma$ evaluated so far. The goal is to provide an estimate $\hat{f}_{j}$ of $f_{j}$ for each item $j \in[n]$.

Cormode and Muthukrishnan have introduced in [8] the CounT-Min sketch that provides, for each item $j$ an $(\varepsilon, \delta)$ additive-approximation $\hat{f}_{j}$ of the frequency $f_{j}$. This algorithm leverages collections of 2-universal hash functions. Recall that a collection $\mathbb{H}$ of hash functions $h:[M] \rightarrow\left[M^{\prime}\right]$ is said to be 2-universal if for every 2 distinct items $x, y \in[M]$, $\mathbb{P}_{h \in \mathbb{H}}\{h(x)=h(y)\} \leq \frac{1}{M^{\prime}}$, that is, the collision probability is as if the hash function assigns truly random values to any $x \in[M]$. Carter and Wegman [20] provide an efficient method to build large families of hash functions approximating the 2-universality property.

The VANILla COUNT-MIN sketch consists of a two dimensional count matrix of size $c_{1} \times c_{2}$, where $c_{1}=\left\lceil\log \frac{1}{\delta}\right\rceil$ and $c_{2}=\left\lceil\frac{e}{\varepsilon}\right\rceil$. Each row is associated with a different 2-universal hash function $h_{i}:[n] \rightarrow\left[c_{2}\right]$. When it reads sample $j$, it updates each row: $\forall i \in\left[c_{1}\right]$, count $\left[i, h_{i}(j)\right] \leftarrow \operatorname{count}\left[i, h_{i}(j)\right]+1$. That is, the cell value is the sum of the frequencies of all the items mapped to that cell. Since each row has a different collision pattern, upon request of $\hat{f}_{j^{\prime}}$ we want to return the cell associated with $j^{\prime}$ minimising the collisions impact. In other words, the algorithm returns, as $f_{j^{\prime}}$ estimation, the cell associated with $j^{\prime}$ with the lowest value: $\hat{f}_{j^{\prime}}=\min _{1 \leq i \leq c_{1}}\left\{\operatorname{count}\left[i, h_{i}\left(j^{\prime}\right)\right]\right\}$.

Fed with a stream of $m$ items, the space complexity of this algorithm is $O\left(\frac{1}{\varepsilon} \log \frac{1}{\delta}(\log m+\log n)\right)$ bits, while update and query time complexities are $O(\log 1 / \delta)$. Concerning its accuracy, the following bound holds: $\mathbb{P}\left\{\left|f_{j}-f_{j}\right| \geq \varepsilon(m-\right.$ $\left.\left.f_{j}\right)\right\} \leq \delta$, while $f_{j} \leq \hat{f}_{j}$ is always true.

\section{Windowed COUNT-MiN}

The COUNT-MIN algorithm solves brilliantly the frequency estimation problem. We propose two extensions in order to meet the sliding window model: Proportional and SPLiTTER. Nevertheless, we first introduce two naive algorithms that enjoy optimal bounds with respect to accuracy (algorithm PERFECT) and space complexity (algorithm SIMPLE). Note that in the following $f_{j}$ is redefined as the frequency of item $j$ in the last $N$ samples among the $m$ items of the portion of the stream evaluated so far.

Due to space constraints, some algorithm pseudo-codes and some proofs are available in the companion paper [21], which the interested reader is invited to consult.

\section{A. Perfect Windowed Count-Min}

PERFECT provides the best accuracy by dropping the complexity space requirements: it trivially stores the whole active window in a queue. When it reads sample $j$, it enqueues $j$ and increases all the count matrix cells associated with $j$. Once the queue reaches size $N$, it dequeues the expired sample $j^{\prime}$ and decreases all the cells associated with $j^{\prime}$. The 
frequency estimation is retrieved as in the VANILLA COUNTMIN ( $c f$. Section II-B).

Theorem 3.1: PERFECT is an $(\varepsilon, \delta)$-additive-approximation of the frequency estimation problem in the count-based sliding window model where $\mathbb{P}\left\{\left|\hat{f}_{j}-f_{j}\right| \geq \varepsilon\left(N-f_{j}\right)\right\} \leq \delta$, while $f_{j} \leq \hat{f}_{j}$ is always true.

Theorem 3.2: PERFECT space complexity is $O(N)$ bits, while update and query time complexities are $O(\log 1 / \delta)$.

\section{B. Simple Windowed Count-Min}

SIMPLE is as straightforward as possible and achieves optimal space complexity with respect to the vanilla algorithm. It behaves as the VANILLA COUNT-Min, except that it resets the count matrix at the beginning of each new window. Obviously it provides a really rough estimation since it simply drops all information about any previous window once a new window starts.

Theorem 3.3: SIMPLE space complexity is $O\left(\frac{1}{\varepsilon} \log \frac{1}{\delta}\right.$ $(\log N+\log n))$ bits, while update and query time complexities are $O(\log 1 / \delta)$.

\section{Proportional Windowed Count-Min}

We now present the first extension algorithm, denoted ProportionAl. The intuition behind this algorithm is as follows. At the end of each window, it stores separately a snapshot of the count matrix, which represents what happened during the previous window. Starting from the current count state, for each new sample, it increments the associated cells and decreases all the count matrix cells proportionally to the last snapshot. This smooths the impact of resetting the count matrix throughout the current window.

More formally, after reading $N$ samples, PROPORTIONAL stores the current count matrix and divides each cell by the window size: $\forall i_{1}, i_{2} \in\left[c_{1}\right] \times\left[c_{2}\right]$, snapshot $\left[i_{1}, i_{2}\right] \leftarrow$ count $\left[i_{1}, i_{2}\right] / N$. This snapshot represents the average step increment of the count matrix during the previous window. When PROPORTIONAL reads sample $j$, it increments the count cells associated with $j$ as in the VANILLA COUNTMIN and subtracts snapshot from count: $\forall i_{1}, i_{2} \in\left[c_{1}\right] \times$ $\left[c_{2}\right]$, count $\left[i_{1}, i_{2}\right] \leftarrow \operatorname{count}\left[i_{1}, i_{2}\right]-\operatorname{snapshot}\left[i_{1}, i_{2}\right]$. Finally, the frequency estimation is retrieved from count as in the vanilla algorithm.

Theorem 3.4: PROPORTIONAL space complexity is $O\left(\frac{1}{\varepsilon}\right.$ $\left.\log \frac{1}{\delta}(\log N+\log n)\right)$ bits. Update and query time complexities are $O\left(\frac{1}{\varepsilon} \log 1 / \delta\right)$ and $O(\log 1 / \delta)$.

\section{Splitter Windowed Count-Min}

PROPORTIONAL removes the average frequency distribution of the previous window from the current window. Consequently, PROPORTIONAL does not capture sudden changes in the stream distribution. To cope with this flaw, one could track these critical changes through multiple snapshots. However, each row of the count matrix is associated with a specific 2-universal hash function, thus changes in the stream distribution will not affect equally each rows.

Therefore, SPLITTER proposes a finer grained approach analyzing the update rate of each cell in the count matrix. To

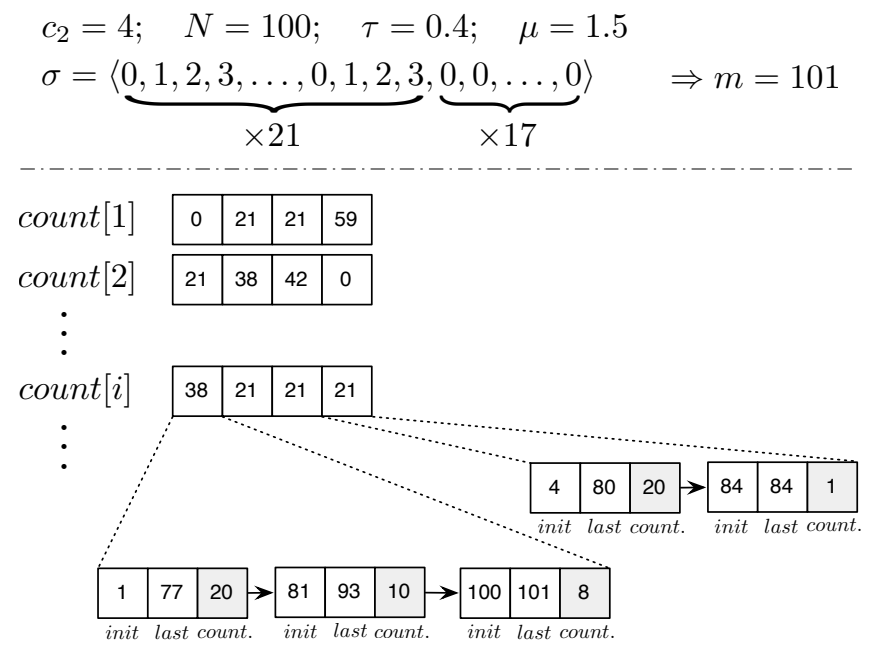

Fig. 1: State of the data structure of SPLITTER after a prefix of 101 items of $\sigma$.

record changes in the cell update rate, we add a (fifo) queue of sub-cells to each cell. When SPLITTER detects a relevant variation in the cell update rate, it creates and enqueues a new sub-cell. This new sub-cell then tracks the current update rate, while the former one stores the previous rate.

Each sub-cell has a frequency counter and 2 timestamps: init, that stores the (logical) time where the sub-cell started to be active, and last, that tracks the time of the last update. After a short bootstrap, any cell contains at least two sub-cells: the current one that depicts what happened in the very recent history, and a predecessor representing what happened in the past. Listing III.1 presents the global behavior of SPLITTER, while Figure 1 illustrates a possible state of the data structure of SPLITTER, after reading a prefix of 101 items of $\sigma$, which is introduced in the top part of the figure with all the parameters of SPLITTER.

SPLITTER spawns additional sub-cells to capture distribution changes. The decision whether to create a new sub-cell is tuned by two parameters, $\tau$ and $\mu$, and an error function: ERROR. Informally, the function ERROR evaluates the potential amount of information lost by merging two consecutive subcells, while $\mu$ represents the amount of affordable information loss. Performing this check at each sample arrival may lead to erratic behaviors. To avoid this, we introduced $\tau$, such that $0<\tau \leq 1$, that sets the minimal length ratio of a sub-cell before taking this sub-cell into account in the decision process.

In more details, when SPLITTER reads sample $j$, it has to phase out the expired data from each sub cell. Then, for each cell of count, it retrieves the oldest sub-cell in the queue, denoted first (Line 9). If first was active precisely $N$ steps ago (Line 10), then it computes the rate at which first has been incremented while it was active (Line 11). This value is subtracted from the cell counter $v$ (Line 12) and from first counter (Line 13). Having retracted what happened $N$ steps ago, first moves forward increasing its init timestamp (Line 14). Finally, first is removed if it has expired (Lines 15 and 16).

The next part handles the update of the cells associated with item $j$. For each of them (Line 19), SPLITTER increases 
Listing III.1: SPLITTER WINDOWEd CounT-MIN 1: init do

2: $\quad$ count $\left[1 \ldots c_{1}\right]\left[1 \ldots c_{2}\right] \leftarrow \overrightarrow{\langle\emptyset, 0\rangle} \quad \triangleright$ the set is a queue

3: Choose $c_{1}$ independent hash functions $h_{1} \ldots h_{c_{1}}$ :

$[n] \rightarrow\left[c_{2}\right]$ from a 2 -universal family.

4: $\quad m^{\prime} \leftarrow 0$

5: end init

upon $\langle$ Sample $\mid j\rangle$ do

for $i_{1}=1$ to $c_{1}$ and $i_{2}=1$ to $c_{2}$ do

$\langle$ queue,$v\rangle \leftarrow$ count $\left[i_{1}, i_{2}\right]$

first $\leftarrow$ head of queue

if $\exists$ first $\wedge$ first init $=m^{\prime}-N$ then

$v^{\prime} \leftarrow \frac{\text { first counter }}{\text { first }_{\text {last }}-\text { firstinit }_{\text {int }}}$

$v \leftarrow v-v^{\prime}$

first founter $_{\text {first }} \leftarrow$ counter $-v^{\prime}$

first $_{\text {init }} \leftarrow$ first $_{\text {init }}+1$

if first $_{\text {init }}>$ first last $_{\text {lat }}$ then

removes first from queue end if

end if

if $h_{i_{1}}(j)=i_{2}$ then

$v \leftarrow v+1$

last $\leftarrow$ bottom of queue

if Alast then

Creates and enqueues a new sub-cell

else if last counter $<\frac{\tau N}{c_{2}}$ then

Updates sub-cell last

else

pred $\leftarrow$ predecessor of last in queue

if $\exists$ pred $\wedge \operatorname{ERROR}($ pred, last $) \leq \mu$ then else

Merges last into pred and renews last end if

end if

end if

end for

count $\left[i_{1}, i_{2}\right] \leftarrow\langle q u e u e, v\rangle$

$m^{\prime} \leftarrow m^{\prime}+1$

end upon

function $\operatorname{GeTFREQ}(j) \quad \triangleright$ returns $\hat{f}_{j}$

return round $\left\{\min \left\{\operatorname{count}[i]\left[h_{i}(j)\right] \cdot v \mid 1 \leq i \leq c_{1}\right\}\right\}$

end function

the cell counter $v$ (Line 20) and retrieves the current sub-cell, denoted last (Line 21). (a) If last does not exist, it creates and enqueues a new sub-cell (Line 23). (b) If last has not reached the minimal size to be evaluated (Line 24), last is updated (Line 25). (c) If not, SPLITTER retrieves the predecessor of last: pred (Line 27). (c.i) If pred exists and the amount of information lost by merging is lower than the threshold $\mu$ (Line 28), SPLITTER merges last into pred and renews last (Line 29). (c.ii) Otherwise it creates and enqueues a new subcell (Line 31), i.e., it splits the cell.

Lemma 3.5: [Number of Splits Upper-bound] Given $0<$ $\tau \leq 1$, the maximum number $\bar{s}$ of splits (number of sub-cells spawned to track distribution changes) is $O\left(\frac{1}{\varepsilon \tau} \log \frac{1}{\delta}\right)$.

Proof: A sub-cell is not involved in the decision process of merging or splitting while its counter is lower than $\frac{\tau N}{c_{2}}=\varepsilon \tau N$. So, no row can own more than $\frac{1}{\varepsilon \tau}$ splits. Thus, the maximum numbers of splits among the whole data structure count is $s=O\left(\frac{1}{\varepsilon \tau} \log \frac{1}{\delta}\right)$.

Theorem 3.6: SPLITTER space complexity is $O\left(\frac{1}{\tau \varepsilon} \log \frac{1}{\delta}\right.$ $(\log N+\log n))$ bits, while update and query time complexities are $O(\log 1 / \delta)$.

Proof: Each cell of the count matrix is composed of a counter and a queue of sub-cells made of two timestamps and a counter, all of size $O(\log N)$ bits $^{2}$. Without any split and considering that all cells have bootstrapped, the initial space complexity is $O\left(\frac{1}{\varepsilon} \log \frac{1}{\delta}(\log N+\log n)\right)$ bits. Each split costs two timestamps and a counter (size of a sub-cell). Let $s$ be the number of splits, we have $O\left(\frac{1}{\varepsilon} \log \frac{1}{\delta}(\log N+\log n)+s \log N\right)$ bits. Lemma 3.5 establishes the following space complexity bound: $O\left(\frac{1}{\varepsilon} \log \frac{1}{\delta}(\log N+\log n)+\frac{1}{\varepsilon \tau} \log \frac{1}{\delta} \log N\right)$ bits.

Each update requires to access each of the count matrix cells in order to move the sliding window forward. However, we can achieve the same result by performing this phase-out operation (from Line 10 to Line 18) only on the count matrix cells that are accessed by the update and query procedures. Given this optimization, update and query require to lookup one cell by row of the count matrix. Then, the query and update time complexities are $O(\log 1 / \delta)$.

One can argue that sub-cell creations and destructions cause memory allocations and disposals. However, we believe that it is possible to avoid wild memory usage leveraging the sub-cell creation patterns, either through a smart memory allocator or a memory aware data structure.

Finally, Table I summarizes the space, update and query complexities of the presented algorithms.

\section{E. Distributed Count-Min}

The functional monitoring model [9] extends the data streaming model by considering a set of $k$ nodes, each receiving an inbound stream $\sigma_{\ell}(\ell \in[k])$. These nodes interact only with a specific node called coordinator.

Notice that the count matrix is a linear-sketch data structure, which means that for every two streams $\sigma_{1}$ and $\sigma_{2}$, we have $\operatorname{CounT-Min}\left(\sigma_{1} \cup \sigma_{2}\right)=\operatorname{Count-Min}\left(\sigma_{1}\right) \oplus \operatorname{Count}-\operatorname{Min}\left(\sigma_{2}\right)$, where $\sigma_{1} \cup \sigma_{2}$ is a stream containing all the samples of $\sigma_{1}$ and $\sigma_{2}$ in any order, and $\oplus$ sums the underlying count matrix term by term. Considering only the last $N$ samples of $\sigma_{1}$ and $\sigma_{2}$, the presented algorithms are also linear-sketches.

The sketch property is suitable for the distributed context. Each node can run locally the algorithm on its own stream $\sigma_{\ell}$ $(\ell \in[k])$. The coordinator can retrieve all the count $_{\ell}$ matrices $(\ell \in[k])$, sum them up and obtain the global matrix $\overline{c o u n t}=$ $\bigoplus_{\ell \in[k]}$ count $_{\ell}$. The coordinator is then able to retrieve the frequency estimation for each item on the global distributed stream $\bar{\sigma}=\sigma_{1} \cup \ldots \cup \sigma_{k}$.

Taking inspiration from [15], we can define the DISTRIBUTED COUNT-MIN (DCM) algorithm, which sends the count matrix to the coordinator each $\varepsilon N$ samples. DCM can be applied to the four aforementioned windowed extensions of VANILla COUNT-Min, resulting in a distributed frequency

\footnotetext{
${ }^{2}$ Note that, for the sake of clarity, timestamps are of size $O(\log m)$ bits in the pseudo-code while counters of size $O(\log N)$ bits are sufficient.
} 
TABLE I: Complexities comparison

\begin{tabular}{|l|l|l|l|}
\hline \multicolumn{1}{|c|}{ Algorithm } & \multicolumn{1}{|c|}{ Space (bits) } & Update time & Query time \\
\hline VANILLA COUNT-MIN [8] & $O\left(\frac{1}{\varepsilon} \log \frac{1}{\delta}(\log m+\log n)\right)$ & $O\left(\log \frac{1}{\delta}\right)$ & $O\left(\log \frac{1}{\delta}\right)$ \\
\hline PERFECT & $O(N)$ & $O\left(\log \frac{1}{\delta}\right)$ & $O\left(\log \frac{1}{\delta}\right)$ \\
SIMPLE & $O\left(\frac{1}{\varepsilon} \log \frac{1}{\delta}(\log N+\log n)\right)$ & $O\left(\log \frac{1}{\delta}\right)$ & $O\left(\log \frac{1}{\delta}\right)$ \\
PROPORTIONAL & $O\left(\frac{1}{\varepsilon} \log \frac{1}{\delta}(\log N+\log n)\right)$ & $O\left(\frac{1}{\varepsilon} \log \frac{1}{\delta}\right)$ & $O\left(\log \frac{1}{\delta}\right)$ \\
SPLITTER & $O\left(\frac{1}{\tau \varepsilon} \log \frac{1}{\delta}(\log N+\log n)\right)$ & $O\left(\log \frac{1}{\delta}\right)$ & $O\left(\log \frac{1}{\delta}\right)$ \\
\hline ECM-SKETCH [17] & $O\left(\frac{1}{\varepsilon^{2}} \log \frac{1}{\delta}\left(\log ^{2} \varepsilon N+\log n\right)\right)$ & $O\left(\log \frac{1}{\delta}\right)$ & $O\left(\log \frac{1}{\delta}\right)$ \\
\hline
\end{tabular}

$(\varepsilon, \delta)$-additive-approximation in the sliding windowed functional monitoring model.

Theorem 3.7: DCM communication complexity is $O\left(\frac{k}{\varepsilon^{2}} \log \frac{1}{\delta} \log N\right)$ bits per window.

Theorem 3.8: DCM introduces an additive error of at most $k \varepsilon N$, i.e, the skew between any cell $\left(i_{1}, i_{2}\right)$ of the global $\overline{\text { count }}$ matrix at the coordinator and the sum of the cells $\left(i_{1}, i_{2}\right)$ of the count $_{\ell}$ matrices $(\ell \in[k])$ on nodes is at most $k \varepsilon N$.

\section{F. Time-based windows}

We have presented the algorithms assuming count-based sliding windows, however all of them can be easily applied to time-based sliding windows. Recall that in time-based sliding windows the steps defining the size of the window are time ticks instead of sample arrivals.

In each algorithm it is possible to split the update code into the subroutine increasing the count matrix and the subroutine phasing out expired data (i.e., decreasing the count matrix). Let denote the former as UPDATESAMPLE and the latter as UPDATETICK. At each sample arrival, the algorithm will perform the UPDATESAMPLE subroutine, while performing the UPDATETICK subroutine at each time tick. Note that timestamps have to be updated using the current time tick count.

This modification affects the complexities of the algorithms, since $N$ is no longer the number of samples, but the number of time ticks. Thus, the complexities improve or worsen, depending if the number of sample arrivals per time tick is greater or lower than 1.

\section{Performance Evaluation}

This section provides the performance evaluation of our algorithms. We have conducted a series of experiments on different types of streams and parameter settings. To verify the robustness of our algorithms, we have fed them with synthetic traces and real-world datasets. The latter give a representation of some existing monitoring applications, while synthetic traces allow to capture phenomena that may be difficult to obtain otherwise. Each run has been executed a hundred times, and we provide the mean over the repeated runs, after removing the 1 st and 10th deciles to avoid outliers.

\section{A. Settings}

If not specified otherwise, in all experiments, the window size is $N=50,000$ and streams are of length $m=3 N$ (i.e. $m=150,000)$ with $n=1,000$ distinct items. Note that we restrict the stream to 3 windows since the behavior of the algorithms in the following windows does not change, as each algorithm relies only on the latest past window. We skip the first window where all algorithms are trivially perfect.

The VANilla CounT-Min uses two parameters: $\delta$ that sets the number of rows $c_{1}$, and $\varepsilon$, which tunes the number of columns $c_{2}$. In all simulations, we have set $\varepsilon=0.1$, meaning $c_{2}=\left\lceil\frac{e}{0.1}\right\rceil=28$ columns. Most of the time, the count matrix has several rows. However, analyzing results using multiple rows requires taking into account the interaction between the hash functions. If not specified, for the sake of clarity, we present the results for a single row $(\delta=0.5)$.

In order to simulate changes in the distribution over time, our stream generator considers a period $p$, a width $w$ and a number of shifts $r$ as parameters. After every $p$ samples, the distribution is shifted right (from lower to greater items) by $w$ positions. Then, after $r$ shifts, the distribution is reset to the initial unshifted version. If not specified, the default settings are $w=2 c_{1}, p=10,000$ and $r=4$.

We evaluate the performance by generating families of synthetic streams, following four distributions: (i) Uniform: uniform distribution; (ii) Normal: truncated standard normal distribution; (iii) Zipf-1: Zipfian distribution with $\alpha=1.0$; and (iv) Zipf-2: Zipfian distribution with $\alpha=2.0$.

We compare SPLITTER with the other presented algorithm, namely PerfeCt SPLitTer and Proportional, as well as with the ECM-SKETCH algorithm proposed by Papapetrou et al. [17].

The wave-based [11] version of ECM-SKETCH that we have implemented replaces each counter of the count matrix with a wave data structure. Each wave is a set of lists, the number and the size of such lists is set by the parameter $\varepsilon_{\text {wave }}$. Then, setting $\varepsilon_{\text {wave }}=\varepsilon$, the wave-based ECM-SKETCH space complexity is $O\left(\frac{1}{\varepsilon} \log \frac{1}{\delta}\left(\frac{1}{\varepsilon} \log ^{2} \varepsilon N+\log n\right)\right)$ bits.

Moreover, recall that SPLITTER has two additional parameters: $\mu$ and $\tau$. We provide the results for $\mu=1.5$ and $\tau=0.05$. Their influence is analyzed separately in Section IV-C. Given these parameters, we have an upper bound of at most $\bar{s}=560$ spawned sub-cells ( $c f$. Lemma 3.5). With the parameters stated so far and the provided memory usage upper bounds, ECMSKETCH uses at least twice the memory required by SPLITTER. Notice however that the upper bound of $\bar{s}=560$ spawned subcells is never reached in any test. Acording to our experiments, ECM-SKETCH uses at least 4.5 times the memory required by SPLITTER in this evaluation.

Finally, the accuracy metric used in our evaluation is the mean absolute error of the frequency estimation of all $n$ items 


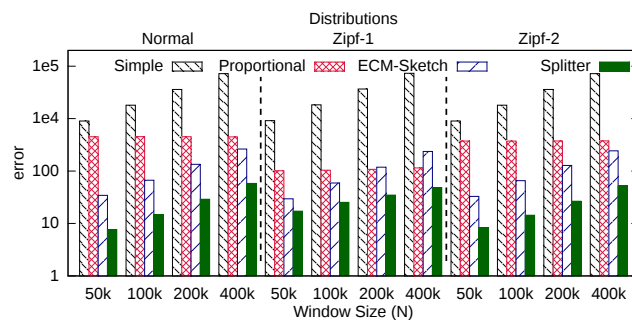

(a) Average estimation error

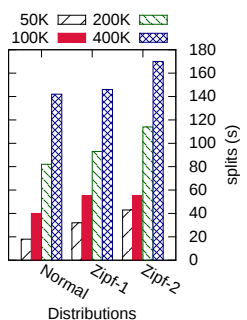

(b) Average splits
Fig. 2: Results for different window sizes $(N)$

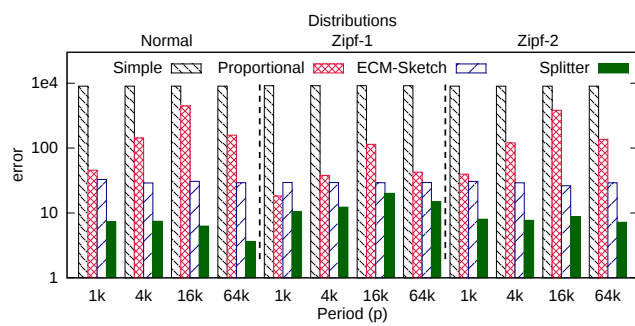

(a) Average estimation error

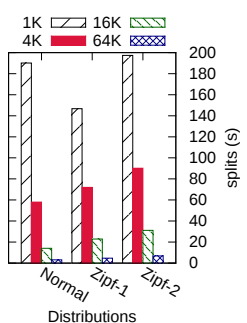

(b) Average splits
Fig. 3: Results for different periods $(p)$

returned by the algorithms with respect to PERFECT, that is $\left(\sum_{j \in[n]}\left|\hat{f}_{j}^{\text {PeRFeCt }}-\hat{f}_{j}^{\text {TestedAlgorithm }}\right|\right) / n$. We refer to this metric as estimation error. We also evaluate the additional space used by SPLiTTER, due to the merge and split mechanisms, through the exact number of splits $s$.

\section{B. Performance comparison}

a) Window sizes: Figure 2(a) presents the estimation error of the Simple, Proportional, Splitter and ECMSKETCH algorithms considering the Normal, Zipf-1 and Zipf-2 distributions, with $N=50,000$ (and a fortiori $m=150,000$ ), $N=100,000$ (with $m=300,000$ ), $N=200,000$ (with $m=600,000$ ) and $N=400,000$ (with $m=1,200,000$ ). Note that the $y$-axis (error) is in logarithmic scale and error values are averaged over the whole stream. SIMPLE is always the worst (with an error equals to 3395 in average), followed by PROPORTIONAL (451 in average), ECM-SKETCH (262 in average) and SPLITTER (57 in average). In average, SPLITTER error is 4 times smaller than ECM-SKETCH, with 4 times less memory requirement. The error estimation of SIMPLE, PROPORTIONAL, ECM-SKETCH and SPLITTER increases in average respectively with a factor $2.0,1.1,1.9$ and 1.7 for each 2 -fold increase of $N$.

Figure 2(b) gives the number of splits spawned by SPLITTER in average to keep up with the distribution changes. The number of splits grows in average with a factor 1.7 for each each 2 -fold increase of $N$. In fact, as $\tau$ is fixed, the minimal size of each sub-cell grows with $N$, and so does the error.

b) Periods: Recall that the distribution is shifted each $p$ samples. The estimation error and the number of splits for $p \in\{1,000 ; 4,000 ; 16,000 ; 64,000\}$ are displayed in Figure 3. Again, SPlitTer (20 at most) is always better than ECMSKETCH (26 at best) achieving roughly a 4 fold improvement. SIMPLE is always the worst (more than 900), followed by

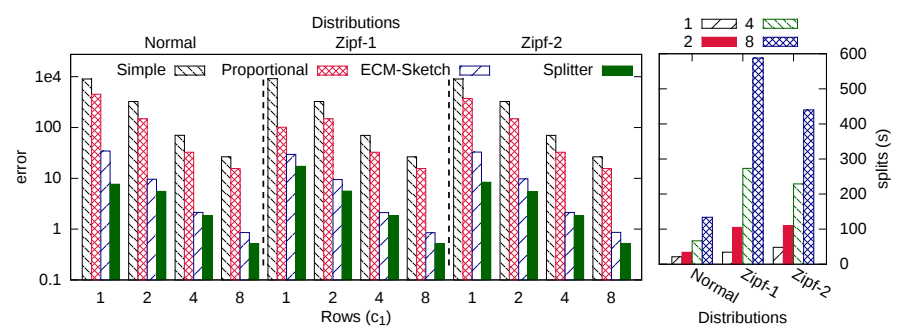

(a) Average estimation error

(b) Average splits

Fig. 4: Results for different number of rows $\left(c_{1}\right)$

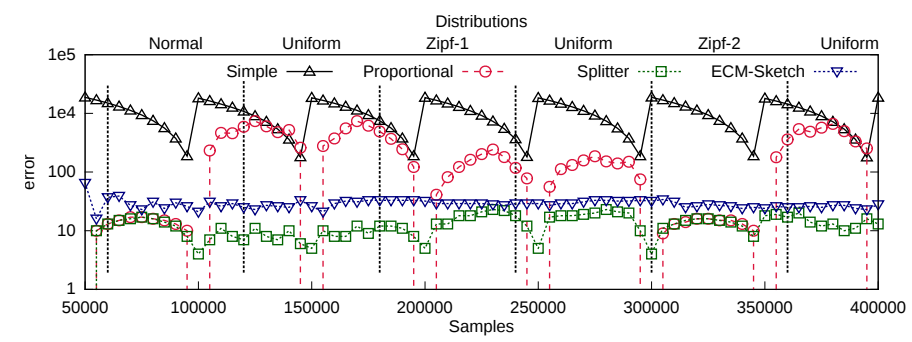

Fig. 5: Estimation error with multiple distributions

PROPORTIONAL (roughly 140 in average). In more details, PROPORTIONAL grows from 1,000 to 16,000 , because slower shifts cast the error on less items, resulting in a larger mean absolute error. However, for 64,000 we have less than a shift per window, meaning that some window will have a non-changing distribution and PROPORTIONAL will be almost perfect. In general SPLITTER estimation error is not heavily affected by decreasing $p$ since it keeps up by spawning more sub-cells. For $p=64,000$ we have at most 7 splits, while for $p=1,000$ we have in average 166 splits. Each 4 -fold decrease of $p$ increases the number of splits by $3.4 \times$ in average.

c) Rows: The Count-Min algorithm uses a hashfunction for each row mapping items to cells. Using multiple rows produces different collisions patterns, increasing the accuracy. Figure 4 presents the estimation error and splits for $c_{1}=1$ (meaning that $\left.\delta=0.5\right), c_{1}=2(\delta=0.25)$, $c_{1}=4(\delta=0.0625)$ and $c_{1}=8$ rows $(\delta=0.004)$. Increasing the number of rows do enhance the accuracy of the algorithms. However, the ordering among the algorithms does not change: Simple, Proportional, ECM-Sketch and SPLiTTER achieve respectively 331, 126, 11 and 4 in average. For each distribution shift, $2 w$ items change their occurrence probability, meaning that (without collisions) most likely $2 w c_{1}$ cells will change their update rate. Since $w=2 c_{1}$, we have $4 c_{1}^{2}$ potential splits per shift. Hopefully, experiments illustrate that the number of splits growth is not quadratic: in average it increases by $2.4 \times$ for each 4 -fold increase of $c_{1}$.

d) Multiple distributions: This test on a synthetic trace has $p=15,000$ and swaps the distribution each 60,000 samples in the following order: Uniform, Normal, Uniform, Zipf-1, Uniform, Zipf-2, Uniform. The streams is of length $m=400,000$. Note that, in order to avoid side effect, the distribution shift and swap periods are not synchronised with the window size $(N=50,000)$.

Figure 5 presents the estimation error evolution as the stream unfolds. SPLITTER error does not exceed 23 (and is 


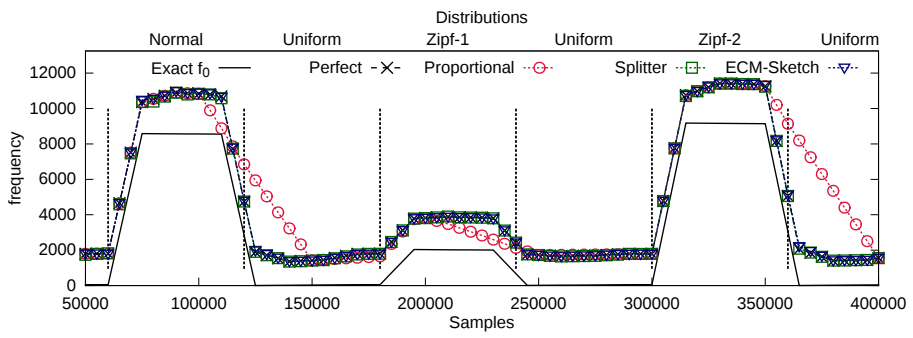

Fig. 6: Estimation of item 0 with multiple distributions

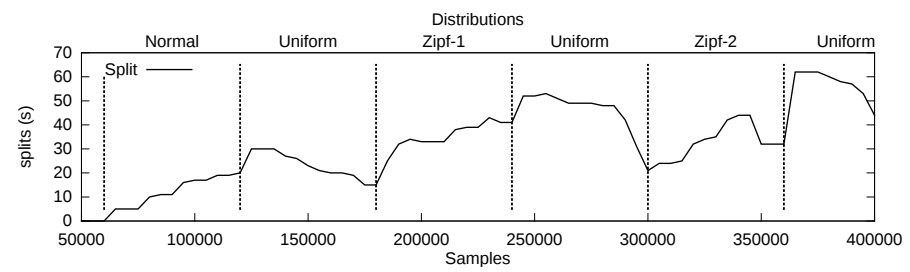

Fig. 7: Number splits $s$ with multiple distributions

equal to 13 in average). ECM-SKETCH maximum error is 65 (29 in average ), as PROPORTIONAL goes up to 740 (207 in average) and SIMPLE reaches 1877 (1035 in average). Since at the beginning of each window SIMPLE resets its count matrix, there is a periodic behavior: the error burst when a window starts and shrinks towards the end. In the 1-st window period (0 to 50,000$)$ and in the 6 -th windows $(250,000$ to 300,000$)$ the distribution does not change over time (shifting Uniform has no effect). This means that SPLITTER does not capture more information than PROPORTIONAL, thus they provide the same estimations in the 2-nd and the 7-th windows (respectively between 50,000 and 100, 000 samples then between 300,000 and 350,000 samples).

Figure 6 presents the value of $f_{0}$ and its estimations over time (for clarity SIMPLE is omitted). The plain line represents the exact value of $f_{0}$ according to time, which also reflects the distribution changes. The plots for PERFECT, ECM-SKETCH and SPLITTER are overlapping (exes, nablas and squares). Except for the error introduced by the CounTMIN approximation, they all follow the $f_{0}$ shape precisely. However, even that is not clearly visible on Figure 6, notice that ECM-SKETCH error is always larger than that of SPLITTER. More precisely, one should observe that item 0 probability of occurrence changes significantly in the following intervals: $[60 k, 75 k],[180 k, 195 k]$ and $[300 k, 315 k]$. PROPORTIONAL fails to follow the $f_{0}$ trend in the windows following those intervals, namely the 3 -rd, 5 -th and 8 -th, since it is unable to correctly assess the previous window distribution.

Finally, Figure 7 presents the number of splits $s$ according to time. There are in average 51 and at most 73 splits (while the theoretical upper bound $\bar{s}$ is 560 according to Lemma 3.5). Interestingly enough, splits decrease when the distribution does not change (in the Uniform intervals for instance). That means that, as expected, some sub-cells expire and no new sub-cells are created. In other words, SPLITTER correctly detects that no changes occur. Conversely, when a distribution shifts or swaps, there is a steep growth, i.e, the change is detected. This pattern is clearly visible in the 2 -nd window.

e) $D D o S$ : As illustrated in the Global Iceberg problem [18], tracking most frequent items in distributed data

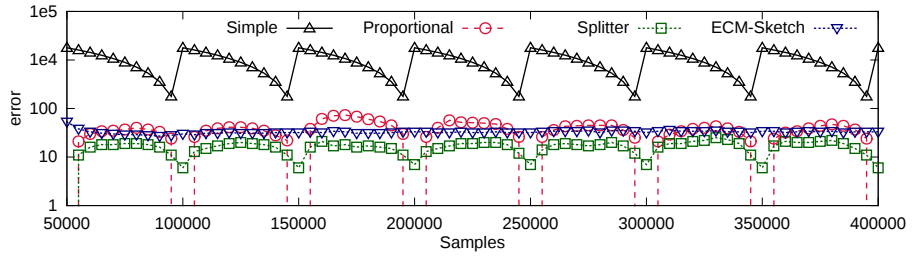

Fig. 8: Results for the DDoS trace

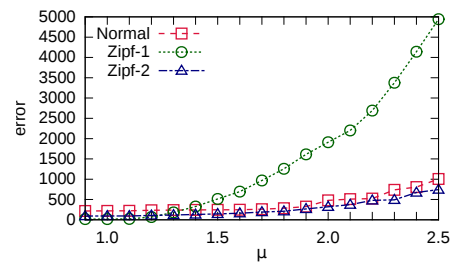

(a) Average estimation error

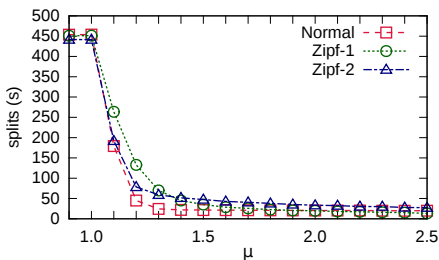

(b) Average number of splits
Fig. 9: Performance comparison with $\tau=0.05$.

streams is not sufficient to detect Distributed Denial of Service (DDoS). As such, one should be able to estimate the frequency of any item. To evaluate our algorithm in this use-case, we have retrieved the CAIDA "DDoS Attack 2007" [22] and "Anonymized Internet Traces 2008" [23] datasets, interleaved them and retained the first 400,000 samples (i.e., the DDoS attack beginning). The stream is composed by $n=4.9 \times 10^{4}$ distinct items. The item representing the DDoS target has a frequency proportion equal to 0.09 , while the second most frequent item owns a 0.004 frequency proportion. Figure 8 presents the estimation error evolution over time. In order to avoid drowning the estimation error in the high number of items, we have restricted the computation to the most frequent 7500 items, which cover $75 \%$ of the stream ${ }^{3}$. Figure 8 illustrates some trends similar to the previous test, however the estimation provided by PROPORTIONAL, ECM-SKETCH and SPLITTER are quite close since the stream changes much less over time. SiMPLE does not make less error than 178 (that is 1002 in average), while PROPORTIONAL, ECM-SKETCH and SPLITTER do not exceed respectively 73 (34 in average), 53 (33 in average) and 25 (16 in average). On the other hand, for SPLITTER, there are at most 154 splits with an average of 105 splits.

\section{Impact of the Splitter parameters}

Figure 9 presents the estimation error and the number of splits with several values of $\mu \in\{0.9,2.5\}$ and a fixed $\tau=$ 0.05 . As expected, the estimation error grows with $\mu$. Zipf-1

\footnotetext{
${ }^{3}$ The remaining items have a frequency proportion lower than $2 \times 10^{-5}$.
}

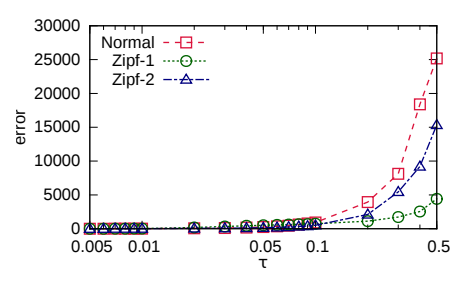

(a) Average estimation error

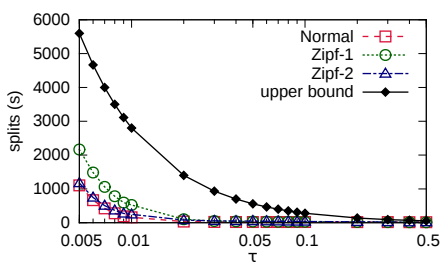

(b) Average number of splits
Fig. 10: Performance comparison with $\mu=1.5$. 
goes from $18(\mu=0.9)$ to $4,944(\mu=2.5)$, while the other distributions in average go from $110(\mu=0.9)$ to $684(\mu=2.5)$. Conversely, increasing $\mu$ decreases the number of splits. Since ERROR cannot return a value lower than 1.0, going from 1.0 to 0.9 has almost no effect with at most 454 splits, which represents roughly $19 \%$ less than the theoretical upper bound. From $\mu=1.0$ to 1.3 , the average falls down to 51 , reaching 20 at $\mu=2.5$. There is an obvious tradeoff around $\mu=1.5$ that should represents a nice parameter choice for a given user.

Figure 10 presents the estimation error and the number of splits according to the parameter $\tau \in\{0.005,0.5\}$, with a fixed $\mu=1.5$. Note that the $x$-axis $(\tau)$ is logarithmic. As for $\mu$, the estimation error increases with $\tau$ : the average starts at 4 (with $\tau=0.005$ ), reaches 610 at $\tau=0.1$ and grows up at 12,198 (for $\tau=0.5$ ). Conversely, increasing $\tau$ decreases the number of splits: the average starts at $1,659(\tau=0.005)$, reaches 77 at $\tau=0.02$ and ends up at $14(\tau=0.5)$. In order to illustrate the accuracy of our splitting heuristic, Figure 10(b) shows also the theoretical upper bound. Again, there seems to be a nice tradeoff around $\tau=0.05$, letting a user having his cake and eat it too!

To summarize, the trend in all the last four plots (and the results for different values of $p$ and $c_{1}$ ) hints to the existence of some optimal value of $\mu$ and $\tau$ that should minimise the error and the splits. This optimal value seems to either be independent from the stream distribution or computed based on the recent behavior of the algorithm and some constraints provided by the user. Seeking for a extensive analysis of this optimum represents a challenging open question.

\section{CONCLUSION AND FUture WORK}

We have presented two $(\varepsilon, \delta)$-additive-approximations for the frequency estimation problem in the sliding windowed data streaming model: PROPORTIONAL and SPLITTER. They have a space complexity of respectively $O\left(\frac{1}{\varepsilon} \log \frac{1}{\delta}(\log N+\log n)\right)$ and $O\left(\frac{1}{\tau \varepsilon} \log \frac{1}{\delta}(\log N+\log n)\right)$ bits, while their update and query time complexities are $O\left(\log \frac{1}{\delta}\right)$.

Leveraging the sketch property, we have shown how to apply our proposal to distributed data streams, with a communication cost of $O\left(\frac{k}{\varepsilon^{2}} \log \frac{1}{\delta} \log N\right)$ bits per window. However, we believe that there is still room for improvement.

We have performed an extensive performance evaluation to compare their respective efficiency and also to compare them to the only similar work in the related works. This study shows the accuracy of both algorithms and that they outperform the only existing solution with real world traces and also with specifically tailored adversarial synthetic traces. Last but not least, these results reach better estimation with respect to the state of the art proposal and required 4 times less memory usage. We have also studied the impact of the two additional parameters of the SPLITTER algorithm $(\tau$ and $\mu)$.

From these results, we are looking forward an extensive formal analysis of the approximation and space bounds of our algorithms. In particular, we seek some insight for computing the optimal values of $\tau$ and $\mu$, minimizing the space usage and maximizing the accuracy of SPLITTER.

\section{REFERENCES}

[1] E. Anceaume and Y. Busnel, "A distributed information divergence estimation over data streams," IEEE Transactions on Parallel and Distributed Systems, vol. 25, 2014.

[2] S. Ganguly, M. Garafalakis, R. Rastogi, and K. Sabnani, "Streaming algorithms for robust, real-time detection of DDoS attacks," in Proceedings of the 27th International Conference on Distributed Computing Systems, ser. ICDCS, 2007.

[3] Z. Bar-Yossef, T. S. Jayram, R. Kumar, D. Sivakumar, and L. Trevisan, "Counting distinct elements in a data stream," in Proceedings of the 6th International Workshop on Randomization and Approximation Techniques, ser. RANDOM, 2002.

[4] D. M. Kane, J. Nelson, and D. P. Woodruff, "An optimal algorithm for the distinct elements problem," in Proceedings of the 19th ACM SIGMODSIGACT-SIGART Symposium on Principles of Database Systems, ser. PODS, 2010.

[5] N. Alon, Y. Matias, and M. Szegedy, "The space complexity of approximating the frequency moments," in Proceedings of the 28th Annual ACM Symposium on Theory of Computing, ser. STOC, 1996.

[6] J. Misra and D. Gries, "Finding Repeated Elements," Science of Computer Programming, vol. 2, 1982.

[7] M. Charikar, K. Chen, and M. Farach-Colton, "Finding frequent items in data streams," in Proceedings of the 29th International Colloquium on Automata, Languages and Programming, ser. ICALP, 2002.

[8] G. Cormode and S. Muthukrishnan, "An improved data stream summary: The count-min sketch and its applications," Journal of Algorithms, vol. 55, 2005.

[9] G. Cormode, S. Muthukrishnan, and K. Yi, "Algorithms for distributed functional monitoring," ACM Transactions on Algorithms, vol. 7, 2011.

[10] M. Datar, A. Gionis, P. Indyk, and R. Motwani, "Maintaining stream statistics over sliding windows," SIAM Journal on Computing, vol. 31, 2002.

[11] P. B. Gibbons and S. Tirthapura, "Distributed streams algorithms for sliding windows," Theory of Computing Systems, vol. 37, 2004.

[12] A. Arasu and G. S. Manku, "Approximate counts and quantiles over sliding windows," in Proceedings of the 23rd ACM SIGMOD-SIGACTSIGART Symposium on Principles of Database Systems, ser. PODS, 2004.

[13] L. Zhang and Y. Guan, "Variance estimation over sliding windows," in Proceedings of the 26h ACM SIGMOD-SIGACT-SIGART Symposium on Principles of Database Systems, ser. PODS, 2007.

[14] L. Golab, D. DeHaan, E. D. Demaine, A. Lopez-Ortiz, and J. I. Munro, "Identifying frequent items in sliding windows over on-line packet streams," in Proceedings of the 3rd ACM SIGCOMM Conference on Internet Measurement, ser. IMC, 2003.

[15] G. Cormode and K. Yi, "Tracking distributed aggregates over time-based sliding windows," in Proceedings of the 24th International Conference on Scientific and Statistical Database Management, ser. SSDBM, 2012.

[16] G. Cormode and S. Muthukrishnan, "What's hot and what's not: tracking most frequent items dynamically," ACM Transactions Database Systems, vol. 30, 2005.

[17] O. Papapetrou, M. N. Garofalakis, and A. Deligiannakis, "Sketch-based querying of distributed sliding-window data streams," Proceedings of the VLDB Endowment, vol. 5, 2012.

[18] E. Anceaume, Y. Busnel, N. Rivetti, and B. Sericola, "Identifying Global Icebergs in Distributed Streams," in Proceedings of the 34th International Symposium on Reliable Distributed Systems, ser. SRDS, 2015.

[19] S. Muthukrishnan, Data streams: algorithms and applications. Now Publishers Inc, 2005.

[20] J. L. Carter and M. N. Wegman, "Universal classes of hash functions," Journal of Computer and System Sciences, vol. 18, 1979.

[21] N. Rivetti, Y. Busnel, and A. Mostefaoui, "Efficiently Summarizing Distributed Data Streams over Sliding Windows," Université de Nantes; Crest(Ensai); Inria Rennes, Tech. Rep. hal-01073877, Jun. 2015.

[22] CAIDA UCSD, “DDoS Attack 2007 dataset," http://www.caida.org/data/ passive/ddos-20070804_dataset.xml, Feb. 2010.

[23] CAIDA UCSD, “Anonymized Internet Traces 2008 dataset," http://www. caida.org/data/passive/passive_2008_dataset.xml, Apr. 2008. 